\title{
A NEW APPROACH ON FUZZY COST-DIFFERENCE RATE COMPARISON IN THE INTEGRATED VENDOR-BUYER PRODUCTION INVENTORY MODEL WITH BACKORDERING
}

\author{
A. Nagoor Gani ${ }^{1}$, S. Raja Dharik ${ }^{2}$ \\ ${ }^{1} \mathrm{PG}$ and Research Department of Mathematics \\ Jamal Mohamed College (Autonomous) \\ Tiruchirappalli, 620020, INDIA \\ ${ }^{2}$ Department of Mathematics \\ Jamal Mohamed College (Autonomous) \\ Tiruchirappalli, 620020, INDIA
}

\begin{abstract}
In today's global markets, an akin co-operative system is essential to cut the joint inventory cost between a vendor and a buyer and also to have a less responding time to the supply chain players. At present, an integrated two-stage production-inventory model have been presented by Yang et al.[15] and the optimal lot size for the vendor and the buyer, also the amount of deliveries in supply chain can be obtained by using the classical differential calculus method. Similarly, several researchers implemented some other algebraic methods to obtain the optimal value for the inventory models. Despite, in these papers the arithmetic-geometric mean inequality and the cost comparisons method do not emphasis on obviously developing the mathematical terms for the two-stage inventory model with backorders. In this observation,
\end{abstract}

\footnotetext{
Received: $\quad$ March 20, 2016

Revised: $\quad$ June 20, 2016

Published: October 21, 2016

(c) 2016 Academic Publications, Ltd. url: www.acadpubl.eu

$\S_{\text {Correspondence author }}$
} 
we develop the inventory model is applied by yang et al.[15] in considering a three-variable inventory problem and presenting an alternate method to establish an ideal replenishment policy for the integrated vendor-buyer inventory model with backordering consuming the fuzzy cost-difference rate comparison approach.

AMS Subject Classification: 03E72, 90B05

Key Words: integrated vendor-buyer inventory, supply chain management, fuzzy costdifference rate comparison, triangular fuzzy number, graded mean integration representation

\section{Introduction}

In the last decade or so, the notion of the vendor-buyer inventory management has gained significent attraction and attention with the helping growth of Supply Chain Management (SCM). Since the trend of the enterprises has been seeking to serve customers better than their competitors in a novel way their products and services, cheaper and faster. It is time for the enterprises managers to understand that they can two more function in aloof. Now, to succeed, it is the need of the hour for the supply chain players to work on a cooperative method in their supply chains with other SC partners. In supply chain to improve competitive advantages, co-operation is one of the important key aspects. Hence, it is inevitable to have coordination with one or two business companies so as to achieve the advantage of competition ie., to increase the revenue and avoid lower supply chain cost [11]. Hence, it is obvious that co-operation and integration are essential tools of supply chain management.

An integrated vendor-buyer inventory system was developed by Yang et al.[15] to derive optimal policy by making use of the ancient differential calculus optimization technique in an environment of with in a just in time (JIT). A lot of algebraic methods like the Cauchy-Bunyakovsky-Schwarz (CBS) inequality, the arithmetic-geometric mean inequality (AGM) etc., are followed in the single or multiple stages of inventory model development by the researchers. Any how, the cost comparison and the two approaches adopted in the research oriented literature. Minnner [7] and Teng [12] developed these methods respectively. In an extensive review [1], we can find that in the derivation of inventory systems, different optimization approaches are used. Despite, the method of cost comparisons does not concentrate on how to develop the mathematical expressions for the two-stage inventory system with the backorders explicitly.

In inventory management, a better cost control of the inventory system is provided by the backordering policy. Relevant to the backordering issues, there 
are many papers dealing with [9] an established optimal policy for deteriorating items with demand of time variation or steady value falling rate or partial exponent backlogging. Assuming that the shortage backorders could be permitted, an optimal EPQ policy with rework and imperfect quality items were derived by Hayek and Salameh [13]. to the deteriorating items with time varying demands and shortages over a finite planning horizon, an inventory problem method was developed by Wang [13] as he considered backlogging rate as time dependent. There are studies which neglect two-stage inventory issue: to evaluate the effect of the postponement strategy on a manufacturer an EPQ based model with planned backorders developed by Li et al.[6]; Wee et al's[4] and Pentico et al's[10] concentration upon an inventory model for items of amelioration and deterioration with partial backordering under inflation; the introduction of the multi-product single-machine production system with stochastic scrapped, partial backordering and service level constraint.

There are two decision variables found in the inventory model [15] of the integrated two-stage vendor-buyer inventory system which can be integer number of $n$, the delivery number and the continuous variable of $Q$, the ordering batch size.

The research takes into account the consideration of the integrated production-inventory model with backordering having three decision variables. Besides, the integer number of $n$, the continuous variable of $Q$ and the backordering rate $K$ are followed in the model development of the two-stage vendor-buyer inventory system. This research differs from the above approach as the Fuzzy cost-difference rate comparison approach to derive and solve the integrated two-stage vendor-buyer inventory system considering backordering is used.

\section{Methodology}

In this section we will review the basic concepts of fuzzy numbers

\subsection{Fuzzy Numbers}

Any fuzzy subset of the real line $\mathbb{R}$, whose membership function $\mu_{A}$ satisfied the following conditions is a generalized fuzzy number $\tilde{A}$.

(i) $\mu_{A}$ is a continuous mapping from $\mathbb{R}$ to the closed interval $[0,1]$;

(ii) $\mu_{A}=0,-\infty<x \leq a_{1}$;

(iii) $\mu_{A}=L(x)$ is strictly increasing on $\left[a_{1}, a_{2}\right]$; 
(iv) $\mu_{A}=\omega_{A}, a_{1} \leq x \leq a_{2}$;

(v) $\mu_{A}=R(x)$ is strictly decreasing on $\left[a_{3}, a_{4}\right]$;

(vi) $\mu_{A}=0, a_{4}<x \leq \infty$.

where $0<\omega_{A} \leq 1$ and $a_{1}, a_{2}, a_{3}$ and $a_{4}$ are real numbers. Also this type of generalized fuzzy number be denoted as $\tilde{A}=\left(a_{1}, a_{2}, a_{3}, a_{4} ; \omega_{A}\right)_{L R}$; when $\omega_{A}=1$, it can be simplified as $\tilde{A}=\left(a_{1}, a_{2}, a_{3}, a_{4}\right)_{L R}$.

\subsection{Triangular Fuzzy Numbers}

The fuzzy set $\tilde{A}=\left(a_{1}, a_{2}, a_{3}\right)$ where $a_{1}<a_{2}<a_{3}$ and defined on $\mathbb{R}$, is called the triangular fuzzy number, if the membership function of $\tilde{A}$ is given by

$$
\mu_{A}= \begin{cases}\frac{x-a_{1}}{a_{2}-a_{1}}, & \text { if } a_{1} \leq x \leq a_{2} \\ \frac{a_{3}-x}{a_{3}-a_{2}}, & \text { if } a_{2} \leq x \leq a_{3} \\ 0, & \text { if otherwise }\end{cases}
$$

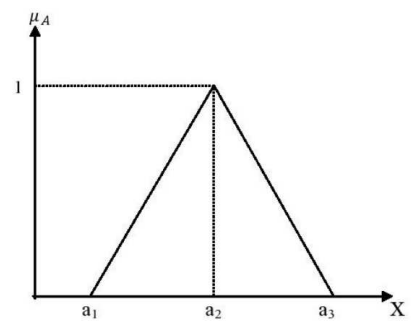

Figure 1: Triangular Fuzzy Number

\subsection{The Function Principle}

The function principle is used for the operation of addition, subtraction, multiplication and division of fuzzy numbers. Suppose $\tilde{A}=\left(a_{1}, a_{2}, a_{3}\right)$ and $\tilde{B}=$ $\left(b_{1}, b_{2}, b_{3}\right)$ are two triangular fuzzy numbers. Then

(i) The addition $\tilde{A}$ and $\tilde{B}$ is $\tilde{A}+\tilde{B}=\left(a_{1}+b_{1}, a_{2}+b_{2}, a_{3}+b_{3}\right)$, where $a_{1}, a_{2}, a_{3}, b_{1}, b_{2}, b_{3}$ are real numbers 
(ii) The multiplication of $\tilde{A}$ and $\tilde{B}$ is $\tilde{A} \times \tilde{B}=\tilde{C}=\left(c_{1}, c_{2}, c_{3}\right)$, where

$$
T=\left\{a_{1} b_{1}, a_{1} b_{3}, a_{3} b_{1}, a_{3} b_{3}\right\}
$$

$c_{1}=\min T, c_{2}=a_{2} b_{2}, c_{3}=\max T$ If $a_{1}, a_{2}, a_{3}, b_{1}, b_{2}, b_{3}$ are all non zero positive real numbers, then $\tilde{A} \times \tilde{B}=\left(a_{1} b_{1}, a_{2} b_{2}, a_{3} b_{3}\right)$

(iii) $-\tilde{B}=\left(-b_{3},-b_{2},-b_{1}\right)$ then the subtraction of $\tilde{A}$ and $\tilde{B}$ is $\tilde{A}-\tilde{B}=\left(a_{1}-\right.$ $\left.b_{3}, a_{2}-b_{2}, a_{3}-b_{1}\right)$ where $a_{1}, a_{2}, a_{3}, b_{1}, b_{2}, b_{3}$ are real numbers

(iv) $\frac{1}{\tilde{B}}=\tilde{B}^{-1}=\left(\frac{1}{b_{3}}, \frac{1}{b_{2}}, \frac{1}{b_{1}}\right)$ where $b_{1}, b_{2}, b_{3}$ are all non zero positive real number, then the division of $\tilde{A}$ and $\tilde{B}$ is $\tilde{A} / \tilde{B}=\left(a_{1} / b_{3}, a_{2} / b_{2}, a_{3} / b_{1}\right)$

(v) For any real number $K, K \tilde{A}=\left(K a_{1}, K a_{2}, K a_{3}\right)$ if $K>0$ $K \tilde{A}=\left(K a_{3}, K a_{2}, K a_{1}\right)$ if $K<0$

\subsection{Graded Mean Integration Representation Method}

If $\tilde{A}=\left(a_{1}, a_{2}, a_{3}, a_{4} ; \omega_{A}\right)_{L R}$ is generalized fuzzy number then the defuzzified value $P(\tilde{A})$ by graded mean integration representation method is given by

$$
P(\tilde{A})=\int_{0}^{\omega_{A}} h\left[\frac{L^{-1}(h)+R^{-1}(h)}{2}\right] d h / \int_{0}^{\omega_{A_{1}}} h d h,
$$

with $0<h \leq \omega_{A}$ and $0<\omega_{A} \leq 1$.

If $\tilde{A}=\left(a_{1}, a_{2}, a_{3}\right)$ is a triangular number then the graded mean integration representation of $\tilde{A}$ by above formula is

$$
P(A)=\frac{\int_{0}^{1} h\left[a_{1}+h\left(a_{2}-a_{1}\right)+a_{3}-h\left(a_{3}-a_{2}\right)\right] d h}{2 \int_{0}^{1} h d h}=\frac{a_{1}+4 a_{2}+a_{3}}{6} .
$$

\section{Determine the Optimal Backordering Rate $\left(b_{*}\right)$, the Optimal Lot Size $\left(q_{*}\right)$, and the Optimal Number of Deliveries $\left(n_{*}\right)$ using the Fuzzy Cost-Difference Rate Comparison Method}

\section{Assumptions:}

The algebraic model for the fuzzy integrated two-stage vendor-buyer inventory model is developed under the following assumptions: 
(a) Fuzzy production and demand rates are constant and $\tilde{p}>\tilde{d}$;

(b) The fuzzy integrated system of single-vendor and single-buyer is considered;

(c) The vendor and the buyer have complete knowledge of each other's information;

(d) The buyer's shortage is allowed;

(e) The fuzzy integrated system does not consider Waiting-in-process (WIP) inventory;

(f) The lead-time of the fuzzy integrated system is known.

\section{Notations:}

The following terminologies are used in developing the fuzzy two-stage vendor-buyer inventory system with backorder:

$q$ - Buyer's lot size per delivery;

$n$ - Number of deliveries from the vendor to the buyer per vendor's replenishment interval;

$n q$ - Vendor's lot size per delivery;

$\tilde{S}$ - Vendor's fuzzy setup cost per setup;

$\tilde{A}$ - Buyer's fuzzy ordering cost per order;

$C_{v}$ - Vendor's unit production cost;

$C_{b}-$ Unit purchase cost paid by the purchaser;

$x_{b}$ - Unit backordering cost of the purchaser;

$\tilde{h}$ - Annual inventory fuzzy carrying cost per dollar invested in stocks;

$\tilde{p}-$ Fuzzy production rate per year, where $\tilde{p}>\tilde{d}$;

$\tilde{d}$ - Fuzzy demand rate per year;

$b$ - The backordering ratio;

$(1--b)$ - The non - backordering ratio;

$\widetilde{T C}(q, n, b)$ - The average integrated total cost of the vendor - buyer inventory model considering backordering over time perid $T$ years;

$P[\widetilde{T C}(q, n, b)]$ - Defuzzified value of $\widetilde{T C}(q, n, b)$.

The following diagram illustrates the fuzzy integrated vendor-buyer inventory model with backorders.

In this paper, we assume a finite planning horizon with length $T$. When the fuzzy demand over this horizon is replenished by $n$ equal batch sizes, $q=\tilde{d} T / n$ and $n \geq 1$, it is obvious that $\tilde{d} T /(n-1)>\tilde{d} T / n>\tilde{d} T /(n+1)$. Assuming that 




Figure 2: Fuzzy integrated vendor-buyer inventory model with backordering

the backordering quantity is $b q$ (Figure2), the following fuzzy total cost of the fuzzy integrated two-stage inventory system considering backorder and $n$ equal batch sizes is developed as:

$$
\begin{aligned}
& \widetilde{T C}(q, n, b)=\{\text { vendor's fuzzy setup cost+fuzzy carrying cost }\} \\
& +\{\text { buyer's fuzzy ordering cost+fuzzy carrying cost }\} \\
& \quad+\text { buyer's backordering cost. } \\
& \widetilde{T C}(q, n, b)=\left\{\frac{\tilde{d} \tilde{S}}{n q}+\frac{\tilde{h} q C_{v}}{2}[(n-1)(1-\tilde{d} / \tilde{p})+\tilde{d} / \tilde{p}]\right\} \\
& +\left\{\frac{\tilde{d} \tilde{A}}{q}+\frac{\tilde{h}(1-b)^{2} q C_{b}}{2}\right\}+\frac{b^{2} q x_{b}}{2} .
\end{aligned}
$$

The vendor's average inventory level $\widetilde{I_{v}}$ is derived as follows:

$\widetilde{I_{v}}=$ vendor's time-weighted inventory $/ \mathrm{T}$

=area of Trapezoidal - area of the bold line

$=$ area of Trapezoidal - the sum of the rectangular area $i$, $i=1,2, \ldots, n-1$ 


$$
\begin{aligned}
& \widetilde{I}_{v}=\left\{\frac{\left(2 T_{2}+T_{1}\right) n q}{2}-\frac{q}{\tilde{d}}[q+2 q+\cdots+(n-1) q]\right\} \times \frac{1}{T} \\
& =\left\{\frac{\left(2 T_{2}+T_{1}\right) n q}{2}-\frac{q^{2}}{\tilde{d}} \frac{n(n-1)}{2}\right\} \times \frac{1}{T} \\
& \text { where } T_{1}=\frac{n q}{\tilde{p}}, T_{2}=(n-1) q\left(\frac{1}{\tilde{d}}-\frac{1}{\tilde{p}}\right) \\
& =\left\{\left[2(n-1)\left(\frac{\tilde{p}-\tilde{d}}{\tilde{d} \tilde{p}}\right)+\frac{n}{\tilde{p}}\right] \times \frac{n q^{2}}{2}-\frac{n q^{2}}{2 \tilde{d}}(n-1)\right\} \times \frac{\tilde{d}}{n q} \\
& =\frac{q}{2}\left[(n-1)\left(1-\frac{\tilde{d}}{\tilde{p}}\right)+\frac{\tilde{d}}{\tilde{p}}\right]
\end{aligned}
$$

Consequently, the annual fuzzy carrying cost of the vendor is

$$
\frac{\tilde{h} q C_{v}}{2}\left[(n-1)\left(1-\frac{\tilde{d}}{\tilde{p}}\right)+\frac{\tilde{d}}{\tilde{p}}\right]
$$

Equation (1) can be rearranged as follows:

$$
\widetilde{T C}(q, n, b)=\frac{1}{q}\left\{\tilde{d} \tilde{A}+\frac{\tilde{d} \tilde{S}}{n}\right\}+\frac{q}{2}\left\{\begin{array}{l}
\tilde{h}(1-b)^{2} C_{b}+b^{2} x_{b} \\
+\tilde{h} C_{v}[(n-1)(1-\tilde{d} / \tilde{p})+\tilde{d} / \tilde{b}]
\end{array}\right\}
$$

Consider $\tilde{d}=\left(d_{1}, d_{2}, d_{3}\right), \tilde{p}=\left(p_{1}, p_{2}, p_{3}\right), \tilde{h}=\left(h_{1}, h_{2}, h_{3}\right), \tilde{A}=\left(A_{1}, A_{2}, A_{3}\right)$, and $\tilde{S}=\left(S_{1}, S_{2}, S_{3}\right)$ are triangular fuzzy numbers.

Assume $\tilde{L}=1-\tilde{d} / \tilde{p}$, i.e. $\tilde{L}=\left(L_{1}, L_{2}, L_{3}\right)=\left(1-d_{3} / p_{1}, 1-d_{2} / p_{2}, 1-d_{1} / p_{3}\right)$.

Therefore

$$
\begin{aligned}
& \widetilde{T C}(q, n, b)=\frac{1}{q}\left[\left(d_{1} A_{1}+\frac{d_{1} S_{1}}{n}\right),\left(d_{2} A_{2}+\frac{d_{2} S_{2}}{n}\right),\left(d_{3} A_{3}+\frac{d_{3} S_{3}}{n}\right)\right] \\
& \left.+\frac{q b^{2} x_{b}}{2}+\frac{q}{2}\left[\begin{array}{l}
\left\{h_{1}(1-b)^{2} c_{b}+h_{1} C_{v}\left[(n-1) L_{1}+\frac{d_{1}}{p_{3}}\right]\right. \\
h_{2}(1-b)^{2} c_{b}+h_{2} C_{v}\left[(n-1) L_{2}+\frac{d_{2}}{p_{2}}\right. \\
h_{1}(1-b)^{2} c_{b}+h_{1} C_{v}\left[(n-1) L_{3}+\frac{d_{3}}{p_{1}}\right.
\end{array}\right]\right\}, \\
& P[\widetilde{T C}(q, n, b)]=\frac{1}{6 q}\left[d_{1} A_{1}+\frac{d_{1} S_{1}}{n}+4\left(d_{2} A_{2}+\frac{d_{2} S_{2}}{n}\right)+d_{3} A_{3}+\frac{d_{3} S_{3}}{n}\right] \\
& +\frac{q b^{2} x_{b}}{2}+\frac{q}{12}\left[\begin{array}{l}
\left\{h_{1}(1-b)^{2} c_{b}+h_{1} C_{v}\left[(n-1) L_{1}+\frac{d_{1}}{p_{3}}\right]\right\}+ \\
4\left\{h_{2}(1-b)^{2} c_{b}+h_{2} C_{v}\left[(n-1) L_{2}+\frac{d_{2}}{p_{2}}\right]\right\} \\
+\left\{h_{1}(1-b)^{2} c_{b}+h_{1} C_{v}\left[(n-1) L_{3}+\frac{d_{3}}{p_{1}}\right]\right\}
\end{array}\right] \\
& =\frac{1}{6 q}\left[d_{1} A_{1}+4 d_{2} A_{2}+d_{3} A_{3}+\frac{1}{n}\left(d_{1} S_{1}+4 d_{2} S_{2}+d_{3} S_{3}\right)\right] \\
& +\frac{q}{2}\left[b^{2} x_{b}+\frac{C_{b}}{6}(1-b)^{2}\left(h_{1}+4 h_{2}+h_{3}\right)\right. \\
& +\quad \frac{C_{v}}{6}\left\{\begin{array}{l}
h_{1}\left[(n-1) L_{1}+\frac{d_{1}}{p_{3}}\right] \\
+4 h_{2}\left[(n-1) L_{2}+\frac{d_{2}}{p_{2}}\right] \\
+h_{3}\left[(n-1) L_{3}+\frac{d_{3}}{p_{1}}\right]
\end{array}\right\} \mid
\end{aligned}
$$


Therefore

$$
\widetilde{T C}(q, n, b)=\frac{1}{6 q} Y+\frac{q}{2} Z
$$

where:

$$
\begin{gathered}
Y(n)=d_{1} A_{1}+4 d_{2} A_{2}+d_{3} A_{3}+\frac{1}{n}\left(d_{1} S_{1}+4 d_{2} S_{2}+d_{3} S_{3}\right), \\
Z(n, b)=b^{2} x_{b}+\frac{C_{b}}{6}(1-b)^{2}\left(h_{1}+4 h_{2}+h_{3}\right)+\frac{C_{v}}{6}\left\{\begin{array}{c}
h_{1}\left[(n-1) L_{1}+\frac{d_{1}}{p_{3}}\right] \\
+4 h_{2}\left[(n-1) L_{2}+\frac{d_{2}}{p_{2}}\right] \\
+h_{3}\left[(n-1) L_{3}+\frac{d_{3}}{p_{1}}\right]
\end{array}\right\} .
\end{gathered}
$$

Optimal ratio $b^{*}$ by using the Fuzzy cost-difference rate comparison method. In this paper we use an easy approach of the Fuzzy cost-difference rate comparison method which consider the difference of average inventory cost rate per unit time between near optimal solution and optimal solution. For deriving the optimal solution, the Fuzzy cost-difference rate comparison method increases the decision variable by a small step-size value of real number and substitutes the consecutive values of decision variable into the average inventory cost rate till the optimal conditions are satisfied.

To determine the optimal backorder for a given horizon $T$, this paper increases the value of $b$ by a small step size of $\lambda$ until $P[\widetilde{T C}(q, n, b-\lambda)] \geq$ $P[\widetilde{T C}(q, n, b)]$ and $P[\widetilde{T C}(q, n, b+\lambda)] \geq P[\widetilde{T C}(q, n, b)]$ are satisfied simultaneously. The cost differences of inventory cost rate between two consecutive real numbers of $b$ satisfy the following conditions:

$$
\begin{aligned}
& P[\widetilde{T C}(q, n, b+\lambda)]-P[\widetilde{T C}(q, n, b)] \geq 0, \\
& P[\widetilde{T C}(q, n, b-\lambda)]-P[\widetilde{T C}(q, n, b)] \geq 0,
\end{aligned}
$$

where $0<\lambda<1$.

When the horizon $T$ and $n$ tend to infinity, $\lim _{T, n \rightarrow \infty} \frac{n-1}{n}=\lim _{T, n \rightarrow \infty} \frac{n+1}{n}=1$ results in $\lim _{T, n \rightarrow \infty} \frac{\tilde{d} T}{n+1}=\lim _{T, n \rightarrow \infty} \frac{\tilde{d} T}{n}=\lim _{T, n \rightarrow \infty} \frac{\tilde{d} T}{n-1}=q$. Obviously, the minimal total cost rate is dependent on the determination of backordering rate $b$. After some algebraic expression we get

$$
\begin{aligned}
& P[\widetilde{T C}(q, n, b+\lambda)]-P[\widetilde{T C}(q, n, b)]=\frac{\lambda+2 b}{2} \geq \frac{C_{b}\left(h_{1}+4 h_{2}+h_{3}\right)}{6 x_{b}+C_{b}\left(h_{1}+4 h_{2}+h_{3}\right)}, \\
& P[\widetilde{T C}(q, n, b-\lambda)]-P[\widetilde{T C}(q, n, b)]=\frac{C_{b}\left(h_{1}+4 h_{2}+h_{3}\right)}{6 x_{b}+C_{b}\left(h_{1}+4 h_{2}+h_{3}\right)} \geq \frac{2 b-\lambda}{2} .
\end{aligned}
$$


Then

$$
\frac{\lambda+2 b}{2} \geq \frac{C_{b}\left(h_{1}+4 h_{2}+h_{3}\right)}{6 x_{b}+C_{b}\left(h_{1}+4 h_{2}+h_{3}\right)} \geq \frac{2 b-\lambda}{2} .
$$

From (3), when $\lambda$ approximates to zero, using the result of Sandwich theorem we have

$$
\lim _{\lambda \rightarrow 0} \frac{\lambda+2 b}{2}=b \geq \frac{C_{b}\left(h_{1}+4 h_{2}+h_{3}\right)}{6 x_{b}+C_{b}\left(h_{1}+4 h_{2}+h_{3}\right)} \geq \lim _{\lambda \rightarrow 0} \frac{2 b-\lambda}{2}=b .
$$

Then

$$
b^{*}=\frac{C_{b}\left(h_{1}+4 h_{2}+h_{3}\right)}{6 x_{b}+C_{b}\left(h_{1}+4 h_{2}+h_{3}\right)} .
$$

Substituting (4) in (2) we have the total integrated inventory cost is

$$
P[\widetilde{T C}(q, n)]=\frac{1}{6 q} Y(n)+\frac{q}{2} W(n)
$$

where $Y(n)=\left(d_{1} A_{1}+4 d_{2} A_{2}+d_{3} A_{3}\right)+\frac{1}{n}\left(d_{1} S_{1}+4 d_{2} S_{2}+d_{3} S_{3}\right)$,

$$
W(n)=\frac{C_{b} x_{b}\left(h_{1}+4 h_{2}+h_{3}\right)}{6 x_{b}+C_{b}\left(h_{1}+4 h_{2}+h_{3}\right)}+\frac{C_{v}}{6}\left\{\begin{array}{c}
h_{1}\left[(n-1) L_{1}+\frac{d_{1}}{p_{3}}\right] \\
+4 h_{2}\left[(n-1) L_{2}+\frac{d_{2}}{p_{2}}\right] \\
+h_{3}\left[(n-1) L_{3}+\frac{d_{3}}{p_{1}}\right]
\end{array}\right\} .
$$

Optimizing $q^{*}$ by utilizing the Fuzzy cost-difference rate comparison method. For given horizon $T, n$ and $b$, to determine the optimal batch size $q^{*}$, we increase the value of $q$ by a small step size $\mu$ until $P[\widetilde{T C}(q-\mu, n)] \geq P[\widetilde{T C}(q, n)]$ and $P[\widetilde{T C}(q+\mu, n)] \geq P[\widetilde{T C}(q, n)]$ are satisfied simultaneously. Considering to Equation (10), the cost differences of inventory cost rate between two consecutive real numbers of $b$ satisfy the following conditions:

$$
\begin{aligned}
& P[\widetilde{T C}(q-\mu, n)]-P[\widetilde{T C}(q, n)] \geq 0, \\
& P[\widetilde{T C}(q+\mu, n)]-P \widetilde{T C}(q, n)] \geq 0,
\end{aligned}
$$

where $0<\mu<1$.

Applying the optimal conditions, we have

$$
\begin{gathered}
P[\widetilde{T C}(q+\mu, n)]-P[\widetilde{T C}(q, n)]=\frac{W}{2}-\frac{Y}{6 q(q+\mu)} \geq 0, \\
P[\widetilde{T C}(q-\mu, n)]-P \widetilde{T C}(q, n)]=-\frac{W}{2}+\frac{Y}{6 q(q+\mu)} \geq 0 .
\end{gathered}
$$

Then

$$
q(q-\mu) \leq \frac{2 Y}{3 W} \leq q(q+\mu)
$$


From the above inequality, the function of $Y$ and $W$ must be non-negative. It is obvious that $Y>0$ and $[(n-1)(1-\tilde{d} / \tilde{p})+\tilde{d} / \tilde{p}]$ is non-negative. From Equation (6), when $T, n$ and $q$ approach infinity and $\mu$ is small enough, $\lim _{\mu \rightarrow 0, q \rightarrow \infty} \frac{q-\mu}{q}=1=$ $\lim _{\mu \rightarrow 0, q \rightarrow \infty} \frac{q+\mu}{q}$ resulting in $(q-\mu)=q=(q+\mu)$.

Taking limit as $\mu$ tends to zero on both sides of (6), we have

$$
\lim _{\mu \rightarrow 0} q(q-\mu) \cong q^{2} \leq \frac{Y}{3 W} \leq \lim _{\mu \rightarrow 0} q(q+\mu) \cong q^{2} .
$$

Again using the Sandwich theorem, we have $q^{2}=\frac{Y}{3 W}$.

Then solving for $q$, we have the optimal lot size as

$$
q^{*}=\sqrt{\frac{Y}{3 W}}=\sqrt{\frac{d_{1} A_{1}+4 d_{2} A_{2}+d_{3} A_{3}+\frac{1}{n}\left(d_{1} S_{1}+4 d_{2} S_{2}+d_{3} S_{3}\right)}{3 \frac{C_{\mathrm{b}} x_{\mathrm{b}}\left(h_{1}+4 h_{2}+h_{3}\right)}{6 x_{\mathrm{b}}+C_{\mathrm{b}}\left(h_{1}+4 h_{2}+h_{3}\right)}+\frac{C_{\mathrm{v}}}{2}\left\{\begin{array}{c}
h_{1}\left[(n-1) L_{1}+\frac{d_{1}}{p_{3}}\right] \\
+4 h_{2}\left[(n-1) L_{2}+\frac{d_{2}}{p_{2}}\right] \\
+h_{3}\left[(n-1) L_{3}+\frac{d_{3}}{p_{1}}\right]
\end{array}\right\}}} .
$$

Substituting (7) in (2) we have the global minimum of the total cost function is given $n$ is

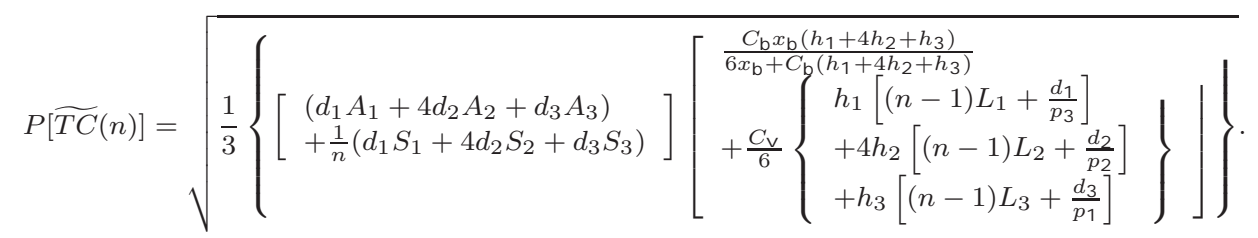

After simplifying we get,

$$
P[\widetilde{T C}(n)]=\sqrt{\frac{1}{3}\{U+V(n)\}}
$$

where the function $V(n)$ is independent of $U$. The function $U$ and $V$ are

$$
\begin{aligned}
U=\frac{C_{b} x_{b}\left(h_{1}+4 h_{2}+h_{3}\right)}{6 x_{b}+C_{b}\left(h_{1}+4 h_{2}+h_{3}\right)}+\frac{C_{v}}{6}\left[\begin{array}{l}
h_{1}\left(\frac{d_{1}}{p_{3}}-L_{1}\right) \\
+4 h_{2}\left(\frac{d_{2}}{p_{2}}-L_{2}\right) \\
+h_{3}\left(\frac{d_{3}}{p_{1}}-L_{3}\right)
\end{array}\right]+\frac{C_{v}}{6}\left(\begin{array}{c}
d_{1} S_{1} \\
+4 d_{2} S_{2} \\
+d_{3} S_{3}
\end{array}\right)\left(\begin{array}{c}
h_{1} L_{1} \\
+4 h_{2} L_{2} \\
+h_{3} L_{3}
\end{array}\right) \\
V(n)=n E+\frac{1}{n} F
\end{aligned}
$$

where $E=\frac{C_{\mathrm{v}}}{6}\left(d_{1} S_{1}+4 d_{2} S_{2}+d_{3} S_{3}\right)\left(h_{1} L_{1}+4 h_{2} L_{2}+h_{3} L_{3}\right)$ and

$$
F=\left(d_{1} S_{1}+4 d_{2} S_{2}+d_{3} S_{3}\right)\left\{\frac{C_{b} x_{b}\left(h_{1}+4 h_{2}+h_{3}\right)}{6 x_{b}+C_{b}\left(h_{1}+4 h_{2}+h_{3}\right)}+\frac{C_{v}}{6}\left[\begin{array}{c}
h_{1}\left(\frac{d_{1}}{p_{3}}-L_{1}\right) \\
+4 h_{2}\left(\frac{d_{2}}{p_{2}}-L_{2}\right) \\
+h_{3}\left(\frac{d_{3}}{p_{1}}-L_{3}\right)
\end{array}\right]\right\} .
$$


To minimize the $P[\widetilde{T C}(n)]$ is equivalent to minimize the function $V(n)$. From $(10)$ to exist, $n E+\frac{F}{n}$ must be positive. since $E$ is positive and $F$ is necessary to be nonnegative. If $F$ is native, it is obvious that the minimum value of (10) can be obtained when $n^{*}=1$.

Optimizing $n^{*}$ by utilizing the Fuzzy cost-difference rate comparison method. Let 1 be the smallest step size instead of a small real numbers, since $n$ is an integer number. For given $q$ and $b$, one can utilize the following optimal conditions of the Fuzzy cost-difference rate comparison method to solve the optimal $n$. The cost differences of cost rate, $V(n)$ between two consecutive integer numbers of $n$ the following conditions:

$$
\begin{aligned}
& V(n+1)-V(n) \geq 0, \\
& V(n+1)+V(n) \geq 0 .
\end{aligned}
$$

Applying the optimal conditions, we have

$$
\begin{gathered}
V(n+1)-V(n)=E-\frac{F}{n(n+1)} \geq 0, \\
V(n-1)-V(n)=-E+\frac{F}{n(n-1)} \geq 0 .
\end{gathered}
$$

Then

$$
n(n-1) \leq \frac{F}{E} \leq n(n+1),
$$

when $n$ tend to infinity, then $\lim _{n \rightarrow \infty} \frac{n}{n-1}=1$ and $\lim _{n \rightarrow \infty} \frac{n}{n+1}=1$ result in $(n-1)=n=$ $(n+1)$. Considering (11), the optimal conditions of $n$ can be written as

$$
\lim _{n \rightarrow \infty} n(n-1) \cong n^{2} \leq \frac{F}{E} \leq \lim _{n \rightarrow \infty} n(n+1) \cong n^{2} .
$$

Again using the Sandwich theorem, we have $n^{2}=\frac{F}{E}$.

We get the optimal $n^{*}$ as

$$
n^{*}=\sqrt{\frac{F}{E}}=\sqrt{\frac{\left(d_{1} S_{1}+4 d_{2} S_{2}+d_{3} S_{3}\right)\left\{\frac{C_{\mathrm{b}} x_{\mathrm{b}}\left(h_{1}+4 h_{2}+h_{3}\right)}{6 x_{\mathrm{b}}+C_{\mathrm{b}}\left(h_{1}+4 h_{2}+h_{3}\right)}+\frac{C_{\mathrm{V}}}{6}\left[\begin{array}{c}
h_{1}\left(\frac{d_{1}}{p_{3}}-L_{1}\right) \\
+4 h_{2}\left(\frac{d_{2}}{p_{2}}-L_{2}\right) \\
+h_{3}\left(\frac{d_{3}}{p_{1}}-L_{3}\right)
\end{array}\right]\right\}}{\frac{C_{V}}{6}\left(d_{1} S_{1}+4 d_{2} S_{2}+d_{3} S_{3}\right)\left(h_{1} L_{1}+4 h_{2} L_{2}+h_{3} L_{3}\right)} .}
$$

However, $n^{*}$ is an integer number. By taking account of the unique positive solution for above quadratic equations on $n^{*}$ in (11), We have

$$
\left(n-\frac{1}{2}\right)^{2} \leq \frac{1}{4}+\frac{F}{E} \leq\left(n+\frac{1}{2}\right)^{2} .
$$

Taking square root and simplifying the above inequality we receive

$$
n^{*} \leq\left(\frac{1}{2}+\sqrt{\frac{1}{4}+\frac{F}{E}}\right) \leq\left(n^{*}+1\right) .
$$


From the above mathematical expression, we get the optimal $n^{*}$ by considering whether $\left(\frac{1}{2}+\sqrt{\frac{1}{4}+\frac{F}{E}}\right)$ is an integer number or not. The following two conditions are used to determine the optimal $n^{*}$.

i If $\left(\frac{1}{2}+\sqrt{\frac{1}{4}+\frac{F}{E}}\right)$ is not an integer number and if the volume of $\left\lfloor\left(\frac{1}{2}+\sqrt{\frac{1}{4}+\frac{F}{E}}\right)\right\rfloor$ equals integer $n$, then the optimal solution is

$$
n^{*}=\left\lfloor\left(\frac{1}{2}+\sqrt{\frac{1}{4}+\frac{F}{E}}\right)\right\rfloor
$$

ii If $\left(\frac{1}{2}+\sqrt{\frac{1}{4}+\frac{F}{E}}\right)$ is an integer number and if the volume of

$\left\lceil\left(\frac{1}{2}+\sqrt{\frac{1}{4}+\frac{F}{E}}\right)\right\rceil$ equals integer $n$, then the optimal solution is $n^{*}=\left\lceil\left(\frac{1}{2}+\sqrt{\frac{1}{4}+\frac{F}{E}}\right)\right\rceil$ and

$$
n^{*}=\left\lceil\left(\frac{1}{2}+\sqrt{\frac{1}{4}+\frac{F}{E}}\right)\right\rceil-1
$$

\section{Algorithm.}

Step 1: Determine $b=\frac{C_{\mathrm{b}}\left(h_{1}+4 h_{2}+h_{3}\right)}{6 x_{\mathrm{b}}+C_{\mathrm{b}}\left(h_{1}+4 h_{2}+h_{3}\right)}$,

$$
\begin{aligned}
& E=\frac{C_{\mathrm{V}}}{6}\left(d_{1} S_{1}+4 d_{2} S_{2}+d_{3} S_{3}\right)\left(h_{1} L_{1}+4 h_{2} L_{2}+h_{3} L_{3}\right) \text { and } \\
& F=\left(d_{1} S_{1}+4 d_{2} S_{2}+d_{3} S_{3}\right)\left\{\frac{C_{\mathrm{b}} x_{\mathrm{b}}\left(h_{1}+4 h_{2}+h_{3}\right)}{6 x_{\mathrm{b}}+C_{\mathrm{b}}\left(h_{1}+4 h_{2}+h_{3}\right)}+\frac{C_{\mathrm{V}}}{6}\left[\begin{array}{c}
h_{1}\left(\frac{d_{1}}{p_{3}}-L_{1}\right) \\
+4 h_{2}\left(\frac{d_{2}}{p_{2}}-L_{2}\right) \\
+h_{3}\left(\frac{d_{3}}{p_{1}}-L_{3}\right)
\end{array}\right]\right\} .
\end{aligned}
$$

If $F$ is greater than zero, then go to step 2. Otherwise $n^{*}=1$.

Step 2: Determine $n^{*}$

$$
\begin{aligned}
& \text { If }\left(\frac{1}{2}+\sqrt{\frac{1}{4}+\frac{F}{E}}\right) \text { is not an integer then } n^{*}=n=\left\lfloor\left(\frac{1}{2}+\sqrt{\frac{1}{4}+\frac{F}{E}}\right)\right\rfloor . \\
& \text { Otherwise, if }\left(\frac{1}{2}+\sqrt{\frac{1}{4}+\frac{F}{E}}\right) \text { is an integer then } n^{*}=\left\lceil\left(\frac{1}{2}+\sqrt{\frac{1}{4}+\frac{F}{E}}\right)\right] \text { and } n^{*}= \\
& \left\lceil\left(\frac{1}{2}+\sqrt{\frac{1}{4}+\frac{F}{E}}\right)\right\rceil-1 .
\end{aligned}
$$

Step 3: Determine $q$ using (7)

Step 4: Determine the total cost of the inventory system with (8).

\section{Numerical Examples}

Example 1. Consider an fuzzy integrated two-stage inventory model with the following parameters:

$\tilde{d}=(1974,1980,1986)$ units per year, $\tilde{p}=(3950,3960,3970)$ per year, $\tilde{h}=(0.1,0.2,0.3)$ per unit per year, $C_{v}=\$ 20$ per unit, $C_{b}=\$ 28.13$ per unit, $x_{b}=\$ 45.1$ per unit, $A=\$(24,25,26)$ per order, and $S=(4196,4201,4206)$ per setup. 
Step 1: Determine $b=\frac{C_{\mathrm{b}}\left(h_{1}+4 h_{2}+h_{3}\right)}{6 x_{\mathrm{b}}+C_{\mathrm{b}}\left(h_{1}+4 h_{2}+h_{3}\right)}=0.11091$,

$$
\begin{aligned}
& E=\frac{C_{\mathrm{V}}}{6}\left(d_{1} S_{1}+4 d_{2} S_{2}+d_{3} S_{3}\right)\left(h_{1} L_{1}+4 h_{2} L_{2}+h_{3} L_{3}\right)=594571.25 \text { and } \\
& F=\left(d_{1} S_{1}+4 d_{2} S_{2}+d_{3} S_{3}\right)\left\{\frac{C_{\mathrm{b}} x_{\mathrm{b}}\left(h_{1}+4 h_{2}+h_{3}\right)}{6 x_{\mathrm{b}}+C_{\mathrm{b}}\left(h_{1}+4 h_{2}+h_{3}\right)}+\frac{C_{\mathrm{V}}}{6}\left[\begin{array}{c}
h_{1}\left(\frac{d_{1}}{p_{3}}-L_{1}\right) \\
+4 h_{2}\left(\frac{d_{2}}{p_{2}}-L_{2}\right) \\
+h_{3}\left(\frac{d_{3}}{p_{1}}-L_{3}\right)
\end{array}\right]\right\} \\
& =249641578.9>0 .
\end{aligned}
$$

Step 2: Determine $n^{*}$

Since $\left(\frac{1}{2}+\sqrt{\frac{1}{4}+\frac{F}{E}}\right)=21$ deliveries, for optimal $n: n^{*}=21$ deliveries and $n^{*}=$ $\left\lceil\left(\frac{1}{2}+\sqrt{\frac{1}{4}+\frac{F}{E}}\right)\right\rceil-1=20$ deliveries.

Therefore, there are two solutions for $n: n^{*}=21$ and $n^{*}=20$.

Step 3: Determine $q^{*}$

$q^{*}(n=21)=137.641$

$q^{*}(n=20)=143.759$

Step 4: Determine the total cost of the inventory system with Equation (8).

Solution 1. $q^{*}=137.641, n=21, b=0.11091$

$P\left[\widetilde{T C}\left(q^{*}=137.641, n=21, b=0.11091\right)\right]=6474.75077$

In this solution, the optimal value of vendor's lot size is of $n q=2890.469$

Solution 2. $q^{*}=143.759, n=20, b=0.11091$

$P\left[\widetilde{T C}\left(q^{*}=143.759, n=20, b=0.11091\right)\right]=6474.74597$

In this solution, the optimal value of vendor's lot size is of $n q=2875.18$

Example 2. Consider the Example 1 with the changes: an integrated two-stage inventory model with the following parameters:

$C_{v}=\$ 30$ per unit, $C_{b}=\$ 33$ per unit, $x_{b}=\$ 40$ per unit, $A=\$(43,45,47)$ per order, and $S=(3895,3900,3905)$ per setup.

Step 1: Determine $b=\frac{C_{\mathrm{b}}\left(h_{1}+4 h_{2}+h_{3}\right)}{6 x_{\mathrm{b}}+C_{\mathrm{b}}\left(h_{1}+4 h_{2}+h_{3}\right)}=0.14163$,

$$
\begin{aligned}
& E=\frac{C_{\mathrm{V}}}{6}\left(d_{1} S_{1}+4 d_{2} S_{2}+d_{3} S_{3}\right)\left(h_{1} L_{1}+4 h_{2} L_{2}+h_{3} L_{3}\right)=1605349.6 \text { and } \\
& F=\left(d_{1} S_{1}+4 d_{2} S_{2}+d_{3} S_{3}\right)\left\{\frac{C_{\mathrm{b}} x_{\mathrm{b}}\left(h_{1}+4 h_{2}+h_{3}\right)}{6 x_{\mathrm{b}}+C_{\mathrm{b}}\left(h_{1}+4 h_{2}+h_{3}\right)}+\frac{C_{\mathrm{V}}}{6}\left[\begin{array}{c}
h_{1}\left(\frac{d_{1}}{p_{3}}-L_{1}\right) \\
+4 h_{2}\left(\frac{d_{2}}{p_{2}}-L_{2}\right) \\
+h_{3}\left(\frac{d_{3}}{p_{1}}-L_{3}\right)
\end{array}\right]\right\} \\
& =262483356.7>0 .
\end{aligned}
$$

Step 2: Determine $n^{*}$

Since $\left(\frac{1}{2}+\sqrt{\frac{1}{4}+\frac{F}{E}}\right)=13.29$ deliveries, $n^{*}=n=\left\lfloor\left(\frac{1}{2}+\sqrt{\frac{1}{4}+\frac{F}{E}}\right)\right\rfloor=13$ deliveries.

Therefore, there is one solution for $n: n^{*}=13$.

Step 3: Determine $q^{*}$

$q^{*}(n=13)=174.8232$

Step 4: Determine the total cost of the inventory system with Equation (8). 
Solution $q^{*}=174.8232, n=13, b=0.14163$

$P\left[\widetilde{T C}\left(q^{*}=174.8232, n=13, b=0.14163\right)\right]=7814.80623$

In this solution, the optimal value of vendor's lot size is of $n q=2272.702$

\section{References}

[1] Cardenas-Barron, L.E., The derivation of EOQ/EPQ inventory models with two backorders costs using analytic geometry and algebra, Appl.Math.Model. 35 (2011) 2394-2407.

[2] Chang, H.C., An application of fuzzy sets theory to the EOQ model with imperfect quality items, Computer and Oper. Res. 31(12) (2004) 2079-2092.

[3] Chen, S.H., Operations on Fuzzy numbers with function principle, Tamkang Journal of Management and Sciences 6(1) (1985) 13-26.

[4] Chen, S.H. and Hsieh, C.H., Graded Mean integration Representation of generalized fuzzy numbers, Journal of Chinese Fuzzy Systems 5(2) (1999) 1-7.

[5] Hayek, P.A. and Salameh, M.K., Production lot sizing with the reworking of imperfect quality items produced, Prod.Plan. Control 12 (2001) 584-590.

[6] Li, J., Wang, S. and Cheng, T.C.E., Analysis of postponement strategy by EPQ based models with planned backorders, Omega 36 (2008) 777-788.

[7] Minner, S., A note on how to compute economic order quantities without derivatives by cost comparisons, Int.J.Prod.Econ. 105(1) (2007) 293-296.

[8] Nagoor Gani, A. and Maheswari, S., Economic order quantity for items with imperfect quality where shortages are backordered in fuzzy environment, Advances in Fuzzy Mathematics, 5(2) (2010) 91-100.

[9] Papachristos, S. and Skouri, K., An optimal replenishment policy for deteriorating items with time-varying demand and partial-exponential type backlogging, Oper. Res. Lett. 27 (2000) 175-184.

[10] Pentico, D.W., Drake, M.J. and Toews, C., The deterministic EPQ with partial backordering: A new approach, Omega 37 (2009) 624-636.

[11] Sarmah, S.P., Acharya, D. and Goyal, S.K., Buyer Vendor coordination models in supply chain management, European J. Oper.Res., 175 (2006) 1-15.

[12] Teng, J.T., A simple method to compute economic order quantities, European J.Oper.Res. 198(1) (2009) 351-352.

[13] Wang, S.P., An inventory replenishment policy for deteriorating items with shortage and partial backlogging, Comput.Oper.Res. 29 (2002) 2043-2051.

[14] Wee, H.M., Law, S.T., Yu, J. and Chen, H.C., An inventory model for ameliorating and deteriorating items with partial backordering under inflation, Int.J.Syst.Sci. 39(8) (2008) 801-807.

[15] Yang, P.C., Wee, H.M. and Yang, H.J., Global optimal policy for vendor-buyer integrated inventory system within just in time environment, J. Global Optim., 37 (2007) 505-511.

[16] Yang, P.C. and Wee, H.M., The economic lot size of the integrated vendor-buyer inventory system derived without derivatives, Optimal Control Appl. Methods, 23(3) (2002) 163169. 
